\title{
Analytical approximations of the dispersion relation of a linear chain of metal nanoparticles
}

\author{
Massimiliano Guasoni *, Costantino De Angelis \\ Dipartimento di Ingegneria dell'Informazione and Consorzio Interuniversitario per le Scienze Fisiche della Materia, Università di Brescia, via Branze 38, Brescia 25123, Italy
}

\section{A R T I C L E I N F O}

\section{Article history:}

Received 22 July 2010

Received in revised form 12 October 2010

Accepted 30 November 2010

Available online 14 December 2010

\section{Keywords:}

Surface plasmon polaritons

Chain of metal nanoparticles

Dipole approximation

Subwavelength limit

\begin{abstract}
A B S T R A C T
We find some useful analytical approximations of the dispersion relation of a linear chain of metal nanoparticles in the subwavelength limit where the dipolar approximation can be used. We also approximate the group velocity without a direct estimation of the derivative of the dispersion relation, that carries unavoidable error amplifications. In the end we use these results in order to get some simple recipes that evaluate the sensitivity of the dispersion relation and the propagation losses with respect to the main parameters of the chain.
\end{abstract}

(C) 2010 Elsevier B.V. All rights reserved.

\section{Introduction}

In the last years the subject of light propagation in linear chains of metal nanoparticles by means of surface plasmon polaritons (SPP) has attracted a lot of interest (see as example [1-4]) because of its large range of potential applications, such as the implementation of biological nanosensors (see [5]), the merging of photonic devices to electronic circuits (see [6]) or in the field of optical imaging (see [7]). It is also considered of deep interest for the realization of new generation solar cells (see [8]).

The dispersion relation carries all the important properties of the chain: its imaginary part defines the propagation losses and from the real part the group velocity is calculated. In order to exactly calculate the dispersion relation the generalized Mie theory of Gerardy-Ausloos for a cluster of spheres should be exploited but it involves the calculation and the inversion of an infinite matrix of scattering coefficients ([9]), then it is not feasible. As it is well known, when the radius $r$ of the spheres is sufficiently smaller than the optical wavelength (i.e. in the subwavelength limit), the propagation in the chain can be described by means of a coupled dipolar approximation (CPM) as done in [10] or equivalently by means of the generalized Mie theory of Gerardy-Ausloos in which only the first scattering coefficient $\Delta_{1}$ is relevant, as discussed in [11] and as we assume in this paper.

In the following, we will consider a chain of equidistant nanospheres with a center-to-center spacing $d$, radius $r$ and dielectric

\footnotetext{
* Corresponding author.

E-mail address: massimiliano.guasoni@ing.unibs.it (M. Guasoni).
}

constant $\varepsilon_{S}$, embedded in a host medium with dielectric constant $\varepsilon_{M}$ and lined up along one direction (see Fig. 1). The corresponding dispersion relation for the transverse and longitudinal modes is given respectively by:

$$
\begin{aligned}
& \omega^{2} i\left(L i_{1}\left(e^{i(\omega-k)}\right)+L i_{1}\left(e^{i(\omega+k)}\right)\right)-\omega\left(L i_{2}\left(e^{i(\omega-k)}\right)+L i_{2}\left(e^{i(\omega+k)}\right)\right)- \\
& i\left(L i_{3}\left(e^{i(\omega-k)}\right)+L i_{3}\left(e^{i(\omega+k)}\right)\right)=-\frac{2}{3} \omega^{3}\left(\Delta_{1}^{-1}\right) \\
& \omega\left(L i_{2}\left(e^{i(\omega-k)}\right)+L i_{2}\left(e^{i(\omega+k)}\right)\right)+i\left(L i_{3}\left(e^{i(\omega-k)}\right)+L i_{3}\left(e^{i(\omega+k)}\right)\right) \\
& =-\frac{1}{3} \omega^{3}\left(\Delta_{1}^{-1}\right)
\end{aligned}
$$

where $\omega=k_{M} d$ ( $k_{M}$ is the wave number of the dielectric host) is the normalized pulsation, $k$ is the propagation constant of the mode normalized to $d$ and $L i_{p}(x)$ is the polylogarithm function of order $p$. Let us call respectively $U_{T}(\omega, k)$ and $A_{T}(\omega)$ the LHS and RHS of Eq. (1) and $U_{L}(\omega, k)$ and $A_{L}(\omega)$ the LHS and RHS of Eq. (2). When losses are neglected (they will be discussed in Section 4), in order to find the propagating modes we only need to look for real values of $k$ between $\omega$ and $\pi$ that solve $\operatorname{Im}\left(U_{T, L}(\omega, k)\right)=\operatorname{Im}\left(A_{T, L}(\omega)\right)$ [11]; we then define $F_{T, L}(\omega, k)=\operatorname{Im}\left(U_{T, L}(\omega, k)\right)$ and $\alpha_{T, L}(\omega)=\operatorname{Im}\left(A_{T, L}(\omega)\right)$. Note that, thanks to the normalization, $F_{T}(\omega, k)$ and $F_{L}(\omega, k)$ do not depend on the system parameters. It follows that all system parameters are into $\alpha_{T}(\omega)$ and $\alpha_{L}(\omega)$ : they contain the scattering coefficient $\Delta_{1}$ of the nanospheres (dependent on $r, \epsilon_{M}$ and $\epsilon_{S}$ ) and this is the only parameter one needs to recalculate when changing the particular system under investigation. 


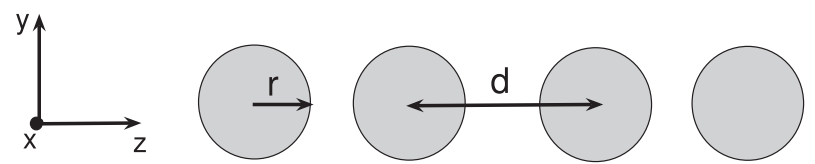

Fig. 1. The chain considered in this paper: the nanospheres have radius $r$ and center-to-center spacing $d$. They have dielectric constant $\varepsilon_{S}$ and are embedded in a host medium with dielectric constant $\epsilon_{M}$.

If we were able to approximate $F_{T}(\omega, k)$ and $F_{L}(\omega, k)$ to get an explicit solution for $k(\omega)$ in Eqs. (1) and (2), we could write $k \approx f$ $(\omega, \alpha)$, with $\alpha$ equal $\alpha_{T}$ for the transverse mode, $\alpha_{L}$ for the longitudinal one. One of the main goals of this paper is thus to find a good $f$ function, i.e. a function as simple as possible and at the same time as accurate as needed. As it will be explained in the last section, these approximations can be translated into simple recipes to guide the design of an optimized chain in the range of frequencies we are interested in.

To reach our goal we use a least-square interpolation (LSI) of $F_{T}(\omega, k)$ and $F_{L}(\omega, k)$ in order to obtain reasonable approximations $\tilde{F}_{T}(\omega, k)$ and $\tilde{F}_{L}(\omega, k)$ in the region $R$ of the plane $(k, w)$ under the light line, i.e. $0 \leq \omega \leq \pi$ and $\omega \leq k \leq \pi$.

In the next sections we discuss this procedure separately for the longitudinal and the transverse modes and we evaluate the errors introduced by this approach in the solution of the dispersion relation.

\section{The longitudinal mode}

Starting from Eq. (2), we define $L_{p}=\left(L i_{p}\left(e^{i(\omega-k)}\right)+L i_{p}\left(e^{i(\omega+k)}\right)\right)$ $(p=1,2,3)$, so that $F_{L}=\omega \operatorname{Im}\left(L_{2}\right)+\operatorname{Re}\left(L_{3}\right)$. Inside $R$ (our region of interest in the $(k, \omega)$ plane), both $\operatorname{Im}\left(L_{2}\right)$ and $\operatorname{Re}\left(L_{3}\right)$ are well described by parabolic functions of both $k$ and $\omega$; it follows that a good set of interpolating functions for $\operatorname{Im}\left(L_{2}\right)$ and $\operatorname{Re}\left(L_{3}\right)$ is $S=\{1, k$, $\left.k^{2}, \omega, \omega k, \omega k^{2}, \omega^{2}, \omega^{2} k, \omega^{2} k^{2}\right\}$. In order to interpolate properly $F_{L}$ we can then use the set $S_{L}=(S \cup S \times \omega)=\left\{1, k, k^{2}, \omega, \omega k, \omega k^{2}, \omega^{2}\right.$, $\left.\omega^{2} k, \omega^{2} k^{2}, \omega^{3}, \omega^{3} k, \omega^{3} k^{2}\right\}$.

We thus use $F_{L}(\omega, k) \approx \tilde{F}_{L}(\omega, k)=k^{2} f_{1}(\omega)+k f_{2}(\omega)+f_{3}(w)$, where $f_{i}(\omega)=a_{i}+b_{i} \omega+c_{i} \omega^{2}+d_{i} \omega^{3}$ with $a_{i}, b_{i}, c_{i}$ and $d_{i}$ the coefficients of the LSI. The inversion of Eq. (2) is now straightforward:

$$
\begin{aligned}
& \tilde{k}(\omega)=-\sqrt{A(\omega) \alpha_{L}(\omega)+B(\omega)}+C(\omega) \\
& A(\omega)=f_{1}^{-1}(\omega) \\
& B(\omega)=-f_{1}^{-1}(\omega) f_{3}(\omega)+(1 / 4) f_{1}^{-2}(\omega) f_{2}^{2}(\omega) \\
& C(\omega)=(-1 / 2) f_{1}^{-1}(\omega) f_{2}(\omega)
\end{aligned}
$$

where $\tilde{k}(\omega)$ is the approximation of $k$; we only consider the solution with the minus sign before the square root because $C(\omega)$ turn out to be always greater than $\pi$ and we use the constraint $\tilde{k}(\omega)<\pi$ for any $\omega$. Note that $A(\omega), B(\omega)$ and $C(\omega)$ do not depend on the particular waveguide parameters so that they need to be calculated only once.

In order to evaluate the validity of the proposed approach the LSI error $E_{L}$ has been computed:

$E_{L}=\frac{\int_{R_{\text {int }}}\left(F_{L}(\omega, k)-\tilde{F}_{L}(\omega, k)\right)^{2}}{\int_{R_{\text {int }}} F_{L}(\omega, k)^{2}}$

For $R_{\text {int }}=R$ in Eq. (4) $E_{L}$ is 5.1 ?\%, but the approximation is not uniform in all $R$, being better in its lower part. In order to improve the accuracy of the approximation, we can then divide $R$ in two parts: $R_{L 1}$ (for $\omega<2.0$ ) and $R_{L 2}$ (for $2.0 \leq \omega \leq \pi$ ) as in Fig. 2). The LSI error then becomes $E_{L 1}=3.1 \%$ in $R_{L 1}$ (Eq. (4) with $R_{i n t}=R_{L 1}$ ) and $E_{L 2}=1.8 \%$ in $R_{L 2}$ (Eq. (4) with $R_{\text {int }}=R_{L 2}$ ).

Another important parameter of the system is the group velocity $g v=\delta \omega / \delta k$. A direct estimation $\left(\tilde{g} v_{D}=1 /(\delta \tilde{k} / \delta \omega)\right)$ requires to take
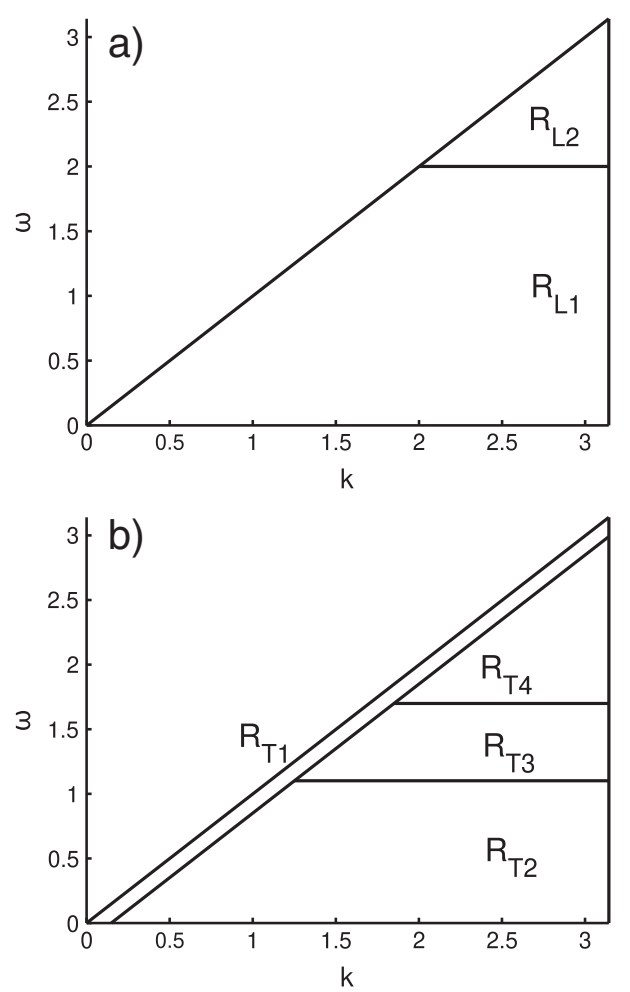

Fig. 2. The region $R$ and its subregions. For the longitudinal case (a) there are the subregions $R_{L 1}([0 \leq \omega \leq 2.0],[\omega \leq k \leq \pi])$ and $\left.R_{L 2}(2.0 \leq \omega \leq \pi],[\omega \leq k \leq \pi]\right)$; for the transverse case (b) there are the subregions $R_{T 1}([0 \leq \omega \leq \pi],[\omega \leq k \leq \omega+0.15]), R_{T 2}$ $([0 \leq \omega \leq 1.1],[\omega+0.15 \leq k \leq \pi]), R_{T 3}([1.1 \leq \omega \leq 1.7],[\omega+0.15 \leq k \leq \pi])$ and $R_{T 4}$ $([1.7 \leq \omega \leq \pi],[\omega+0.15 \leq k \leq \pi])$

a derivative of $\tilde{k}$ with unavoidable error amplifications. A better approach, that does not need to take the derivative of $\tilde{k}$, is to write:

$g v_{I}=\frac{F_{L}^{(k)}(\omega, k)}{\alpha_{L}^{(\omega)}(\omega)-F_{L}^{(\omega)}(\omega, k)}$

where the superscripts $(k)$ and $(\omega)$ indicates the derivative with respect to the variables $k$ and $\omega$ and the subscript $I$ stands for indirect. Note that $g v_{I}=g v_{D}$ as long as we use the exact $k$ value; however, as we will show in detail later on, they are not equivalent if one uses the approximated $\tilde{k}$. We will call indirect estimation $\tilde{g} v_{I}$ of the group velocity the following expression:

$\tilde{g} v_{I}=\frac{F_{L}^{(k)}(\omega, \tilde{k})}{\alpha_{L}^{(\omega)}(\omega)-F_{L}^{(\omega)}(\omega, \tilde{k})}$

Eq. (6) gives an estimation of the group velocity using $\tilde{k}$ calculated with Eq. (3). If $\Delta k$ is the error in the approximation of $k$ (i.e. $\tilde{k}=k+\Delta k)$, then:

$\tilde{g} v_{I}-g v_{I}=g v_{I}(\omega, k+\Delta k)-g v_{I}(\omega, k) \approx \Delta k \frac{\delta g v_{I}}{\delta k}$

So that the error in the estimation of $g v_{I}$ is proportional to $\Delta k$ thus avoiding error amplifications.

\section{The transverse mode}

In the approximation of the dispersion relation of the transverse mode the first problem to face is the presence of the term $\operatorname{Re}\left(L_{1}\right)$ going to infinity along the light line $(\omega=k)$. A possible way out is to consider a subregion $R_{T 1}$ of $\mathrm{R}$ close to the light line such that in $R_{T 1} R e$ 
$\left(L_{1}\right)$ is much greater than $\operatorname{Im}\left(L_{2}\right)$ and $\operatorname{Re}\left(L_{3}\right)$ and in $R-R_{T 1}$ the term $\operatorname{Re}\left(L_{1}\right)$ is sufficiently small.

For example let us choose the subregion $R_{T 1}$ defined by: $0 \leq \omega \leq \pi$ and $\omega \leq k \leq \omega+\epsilon(\epsilon=0.15$ in Fig. 2$)$. In $R_{T 1}$ the main term of $F_{T}(\omega, k)$ is $\omega^{2} \operatorname{Re}\left(L_{1}\right)$ and $\omega \operatorname{Im}\left(L_{2}\right)$ and $\operatorname{Re}\left(L_{3}\right)$ can be accurately approximated by means of a term $f_{1}(\omega)$ that does not depend on $k$. It follows that $F_{T}(\omega, k)$ can be approximated by $\tilde{F}_{T 1}(\omega, k)=\omega^{2} \operatorname{Re}\left(L i_{1}\right)+f_{1}(\omega)$; this makes the inversion of Eq. (1) straightforward by solving for $k$, since $\operatorname{Re}\left(L i_{1}(x)\right)=-\log |2 \sin (x / 2)|$. After some algebra we then get:

$\tilde{k}(\omega)=\operatorname{Arcos}\left(\cos (\omega)-0.5 e^{-\frac{\alpha_{T}(\omega)+f_{1}(\omega)}{\omega^{2}}}\right)$

Function $f_{1}(\omega)$ can be well approximated by $2.292 \cos (1.075 \omega)$ for $\omega \leq 1.7$ and by $\left(-24.599+38.4807 \omega-19.908 \omega^{2}+3.266 \omega^{3}\right)$ for $\omega \geq 1.7$; note that when $\omega$ is small $\alpha_{T}(\omega)+f_{1}(\omega)>>\omega^{2}$, so that from Eq. (8) $\tilde{k}(\omega) \approx \omega$, i.e. in this case the dispersion relation of the transverse mode runs along the light line.

In $R-R_{T 1}$, where the LSI is applied, the contribute of the term $\omega^{2} R e$ $\left(L_{1}\right)$ depends a lot on $\omega$, so that an unique good interpolation is not possible. We then divide $R-R_{T 1}$ in three regions (Fig. 2): $R_{T 2}$ for low values of $\omega(\omega \leq 1.1), R_{T 3}(1.1 \leq \omega \leq 1.7)$ and $R_{T 4}$ for high values of $\omega(\omega \geq 1.7)$.

In $R_{T 2} \operatorname{Re}\left(L_{1}\right)$ has a quasi sinusoidal behaviour, so that a good set of interpolating functions is:

$S_{2}=\{\cos (v(\omega \pm k)), \sin (v(\omega \pm k)), \cos (v k), \sin (v k), \cos (v \omega), \sin (v \omega), 1\}$.

In order to make the inversion of Eq. (1) feasible, we have to use the set $S_{2}$ also for the terms $\operatorname{Im}\left(L_{2}\right)$ and $\operatorname{Re}\left(L_{3}\right)$, so that the complete set we use for the LSI of $F_{T}(\omega, k)$ in $R_{T 2}$ is $S_{T 2}=S_{2} \cup S_{2} \times \omega \cup S_{2} \times \omega^{2}$. The parameter $v$ is used to optimize the interpolation in $R_{T 2}$ and we find that with $v=0.8$ the LSI error (Eq. (4) using $R_{i n t}=R_{T 2}$ and $F_{T}$ instead of $\left.F_{L}\right)$ is minimized and is $3.0 \% . F_{T}(\omega, k)$ is then approximated by $\tilde{F}_{T 2}(\omega, k)=\sin (0.8 k) f_{1}(\omega)+\cos (0.8 k) f_{2}(\omega)+f_{3}(\omega)$, where $f_{i}(\omega)=$ $a_{i}+b_{i} \omega+c_{i} \omega^{2}+d_{i} \sin (0.8 \omega)+e_{i} \cos (0.8 \omega)+f_{i} \omega \sin (0.8 \omega)+g_{i} \omega \cos$ $(0.8 \omega)+h_{i} \omega^{2} \sin (0.8 \omega)+i_{i} \omega^{2} \cos (0.8 \omega)(i=1,2,3)$ and $a_{i}, b_{i}, c_{i}, d_{i}, e_{i}, f_{i}$, $g_{i}, h_{i}, i_{i}$ are the coefficients of the LSI. With some algebra we then get:

$$
\begin{array}{r}
\tilde{k}_{A}(\boldsymbol{\omega})=\frac{1}{0.8}\left[\operatorname{Arcsin}\left(A(\boldsymbol{\omega}) \alpha_{T}(\boldsymbol{\omega})+B(\boldsymbol{\omega})\right)+\operatorname{Arctan}(C(\boldsymbol{\omega}))\right] \\
A(\boldsymbol{\omega})=\left(f_{1}^{2}(\boldsymbol{\omega})+f_{2}^{2}(\boldsymbol{\omega})\right)^{-1 / 2} \\
B(\boldsymbol{\omega})=-f_{3}(\boldsymbol{\omega})\left(f_{1}^{2}(\boldsymbol{\omega})+f_{2}^{2}(\boldsymbol{\omega})\right)^{-1 / 2} \\
C(\boldsymbol{\omega})=-f_{2}(\boldsymbol{\omega}) f_{1}^{-1}(\boldsymbol{\omega})
\end{array}
$$

$\tilde{k}_{B}(\omega)=\frac{1}{0.8}\left[\pi-\operatorname{Arcsin}\left(A(\omega) \alpha_{T}(\omega)+B(\omega)\right)+\operatorname{Arctan}(C(\omega))\right]$

In $R_{T 3} \operatorname{Re}\left(L_{1}\right)$ is well described by the combination of sinusoid and cosinusoid with frequency $v$ and $2 v$, so that a good set of interpolating functions for $\operatorname{Re}\left(L_{1}\right)$ is:

$S_{3}=\{\cos (2 v(\omega \pm k)), \sin (2 v(\omega \pm k)), \cos (2 v k), \sin (2 v k), \cos (2 v \omega), \sin (2 v \omega)$, $\cos (v(\omega \pm k)), \sin (v(\omega \pm k)), \cos (v k), \sin (v k), \cos (v \omega), \sin (v \omega), 1\}$

It follows that the set used for the LSI of $F_{T}(\omega, k)$ in $R_{T 3}$ should be $S_{T 3}=S_{3} \cup S_{3} \times \omega \cup S_{3} \times \omega^{2}$. There are two problems using this last set: first, the inversion of Eq. (1) involves a fourth-degree polynomial equation and moreover the high number of functions makes the correlation matrix of the LSI ill-conditioned. Then we reduce the set and use

$S_{3}=\{\sin (v k) \sin (v \omega), \sin (v k) \cos (2 v \omega), \cos (2 v k) \sin (v \omega), \cos (2 v k) \cos (2 v \omega)$, $\sin (v k), \sin (v \omega), \cos (2 v k), \cos (2 v \omega), 1\}$ with $v=0.5$ that minimize the LSI error (2.8\%), so the interpolation of $F_{T}(\omega, k)$ is $\tilde{F}_{T 3}(\omega, k)=\sin (0.5 k) f_{1}(\omega)+\cos (k) f_{2}(\omega)+f_{3}(\omega)$, where $f_{i}(\omega)=a_{i}+b_{i} \sin (0.5 \omega)+c_{i} \cos (\omega)+d_{i} \omega \sin (0.5 \omega)+e_{i} \omega \cos$ $(\omega)+f_{i} \omega^{2} \sin (0.5 \omega)+g_{i} \omega^{2} \cos (\omega)(i=1,2,3)$ and $a_{i}, b_{i}, c_{i}, d_{i}, e_{i}, f_{i}, g_{i}$ are the coefficients of the LSI. It is now straightforward to obtain:

$$
\begin{aligned}
& \tilde{k}_{A}(\omega)=\frac{1}{0.5} \operatorname{Arcsin}\left( \pm \sqrt{A(\omega) \alpha_{T}(\omega)+B(\omega)}+C(\omega)\right) \\
& A(\omega)=-(1 / 4) f_{2}^{-1}(\omega) \\
& B(\omega)=(1 / 2)+(1 / 2) f_{3}(\omega) f_{2}^{-1}(\omega)+(1 / 16) f_{1}(\omega)^{2} f_{2}^{-2}(\omega) \\
& C(\omega)=(1 / 4)\left(f_{1}(\omega) f_{2}^{-1}(\omega)\right. \\
& \tilde{k}_{B}(\omega)=\frac{1}{0.5}\left[\pi-\operatorname{Arcsin}\left( \pm \sqrt{A(\omega) \alpha_{T}(\omega)+B(\omega)}+C(\omega)\right)\right]
\end{aligned}
$$

As far as subregion $R_{T 4}$ is concerned, here $\operatorname{Re}\left(L i_{1}\right)$ is well described by parabolic functions of both $k$ and $\omega$, so the set $S_{T 4}=S \cup S \times \omega \cup S \times \omega^{2}$ can be used to obtain the interpolation $e F_{T 4}$; the corresponding LSI error is only $1.9 \%$, and then we approximate $k$ with Eq. (3).

As far as the group velocity is concerned, also for the transverse mode the indirect estimation given by Eq. (6) can be used, substituting $\alpha_{L}$ with $\alpha_{T}$ and $F_{L}$ with $F_{T}$.

\section{Results}

The analytical solutions proposed in the previous sections provide a powerful tool to get a deeper insight into the physical properties of the chain. As a reference example in the following we analyze an infinite chain of silver nanospheres embedded in glass $\left(\epsilon_{M}=2.25\right)$ with radius $r=10 \mathrm{~nm}$ and center-to-center spacing $d=25 \mathrm{~nm}$; the dielectric constant $\epsilon_{S}$ of the silver is described by means of Drude's model, for which $\epsilon_{S}(\omega)=1-\left(\omega_{p} d / \omega c\right)^{2}$, where $\omega_{p}$ is the silver plasma frequency (we use $\omega_{p}=10.9 \cdot 10^{15} s^{-1}$ ), $\omega$ is the normalized pulsation and $c$ is the speed of light in the host medium.

We first focus on the dispersion curves and on the group velocities of the modes, then we move on their sensitivity and propagation losses with respect to the parameters of the chain. We also do a comparison with the results obtained by solving numerically Eqs. (1) and (2), postponing in Appendix A a full detailed analysis of the errors committed by using the proposed approximations.

The dispersion curves and the group velocities of the modes are reported in Figs. 3( a), c) and e) ) and 4 (a), c) and e) ), where we can see an optimum agreement between the exact numerical results and our approximated analytical solutions.

By means of a first-order correction, one can prove that a small variation $\Delta \alpha_{T, L}$ of $\alpha_{T, L}$ is responsible for a $k$ variation given by:

$\Delta k=\frac{\Delta \alpha_{T, L}}{F_{T, L}^{(k)}(\omega, k)}$

It can also be proven that in order to calculate the losses $\gamma_{T, L}$ due to the imaginary part of the nanospheres dielectric constant $\varepsilon_{S}$ one can write:

$\gamma_{T, L}=\frac{\operatorname{Re}\left(\Delta A_{T, L}\right)}{F_{T, L}^{(k)}(\omega, k)}$

where $\Delta A_{T, L}$ is the variation of $A_{T, L}$ (due to $\operatorname{Im}\left(\epsilon_{S}\right) \neq 0$ ) defined in section 1 .

Let us now consider the longitudinal mode, for which $F_{L} \approx \tilde{F}_{L}=k^{2} f_{1}(\omega)+k f_{2}(\omega)+f_{3}(\omega)$, so that $F_{L}^{(k)}=2 f_{1}(\omega) k+f_{2}(\omega)=$ $2 f_{1}(\omega)(k-C(\omega))$. Since $C(\omega) \approx \pi, \Delta k$ and $\gamma_{L}$ of Eqs. (13) and (14) are, at first order, proportional to $1 /(k-\pi)$. 
This means that, for a fixed $\omega$, the more $k$ moves away from the light line, the lossier and more sensitive to parameters variations the chain becomes. From Eq. (3) and considering that $f_{1}(\omega)>0$, it is possible to rewrite $F_{L}^{(k)}=-2 f_{1} \sqrt{A \alpha_{L}+B}=-2 \sqrt{f_{1} \alpha_{L}+f_{2}^{2} / 4-f_{3} f_{1}}$. In this way Eqs. (13) and (14) become direct and simple formulas for the sensitivity and the losses that depend on the parameters of the system.

Moreover in the subwavelength limit the scattering coefficient $\Delta_{1}$ (for its formulation see [9]) can be approximated by means of asymptotic formulas for Bessel functions as:

$\Delta_{1} \approx\left[\frac{3 i\left(\epsilon_{S}+2 \epsilon_{M}\right)}{\left(k_{M} r\right)^{3}\left(2 \epsilon_{S}-2 \epsilon_{M}\right)}-1\right]^{-1}$

being $k_{M}=\omega / d$. In this case after some algebra we get:

$\operatorname{Re}\left(A_{L}\right)=\omega^{3} / 3+\operatorname{Im}\left(p_{L}\right) / r^{3}$

$\operatorname{Im}\left(A_{L}\right)=\alpha_{L}=\operatorname{Re}\left(p_{L}\right) / r^{3}$

where $p_{L}=\left[d^{3}\left(\epsilon_{S}+2 \epsilon_{M}\right)\right] /\left[2 r^{3}\left(2 \epsilon_{S}-2 \epsilon_{M}\right)\right]$. When the system is lossless then $\operatorname{Im}\left(\epsilon_{S}\right)=0$, so that $\operatorname{Im}\left(p_{L}\right)=0$ and $\operatorname{Re}\left(A_{L}\right)$ becomes $\omega^{3} / 3$; when absorption is considered, $\operatorname{Im}\left(\epsilon_{S}\right) \neq 0$ and as consequence $\operatorname{Im}$ $\left(p_{L}\right) \neq 0$; it follows that for a fixed $\omega$ the variation $\operatorname{Re}\left(\Delta\left(A_{L}\right)\right)$ due to the absorption is $\operatorname{Im}\left(p_{L}\right) / r^{3}$. It is then possible to rewrite Eq. (14) as:

$\gamma_{L}=\frac{\operatorname{Im}\left(p_{L}\right) / r^{3}}{2 \sqrt{-f_{1} \operatorname{Re}\left(p_{L}\right) / r^{3}+f_{2}^{2} / 4-f_{3} f_{1}}}$

that is the relation between the losses of the longitudinal mode and the parameters of the chain.

It is interesting to note that the equation above is easily invertible in $r$, so that for a fixed $\bar{\gamma}_{L}$ it is possible to find the minimum value of $r$ (once $\omega$ and $\varepsilon_{S}$ are fixed) that makes the propagation losses below $\bar{\gamma}_{L}$.

In order to test the validity of the previous formulas, we introduced a complex dielectric constant $\epsilon_{S}(\omega)=1-\omega_{p}^{2} /[(\omega c / d)(\omega c /$ $d+i T)$ ], using $w_{p}=10.9 \cdot 10^{15} S^{-1}$ and $T=1.6 \cdot 10^{14} S^{-1}$ to take into account the absorption of the metal spheres. In Fig. 5a) we then compared the losses $\gamma_{L}$ calculated as imaginary part of $k$ obtained by numerically solving Eq. (2) with the losses $\gamma_{P L}$ predicted by Eq. (18).
The comparison is done in the band of the longitudinal mode $(0.48<\omega<0.65$, see Fig. $4 a))$ and it is possible to see that they match well: the mean relative error is $6.1 \%$.

Let's note that, as predicted, the more the dispersion curve moves away from the light line, the lossier is the mode and that losses are nearly proportional to $1 /(\pi-k)$ : for example $\gamma_{L}(\omega=0.6) / \gamma_{L}(\omega=0.5)=1.55$ and $(\pi-k(\omega=0.5)) /(\pi-k(\omega=0.6))=1.74$.

In conclusion, the developed approximations are useful in the analysis of nanochains that, as the one considered in this example, can concentrate light in an extremely small sub-wavelength region: in Fig. 5b) the longitudinal mode at $\omega=0.5(\lambda=428 \mathrm{~nm})$ is shown in the transverse plane (finite element simulation) and it can be observed that the energy is quite fully concentrated in a region of $40 \mathrm{~nm}$. This makes the mode to be really potentially useful for nano-optic applications.

Note also that similar considerations can be done for the transverse mode although for the sake of brevity we do no report them here.

\section{Conclusions}

The main goal of this paper has been to find analytical approximations for the dispersion relation $k(\omega)$ and for the group velocity of the propagating modes in a linear chain of metal nanospheres, in order to obtain a deeper insight into its physical properties.

These approximations have been validated by a detailed comparison with the numerical solutions of the exact dispersion relation. Our results proved to be very effective to estimate both wave numbers and group velocities of the modes propagating along the chain. Some examples have been also provided to show how the proposed approximations can be used in order to reveal some important features of the chain; as an example analytical formulas have been derived to evaluate the sensitivity of the dispersion curves and the propagation losses with respect to the parameters of the chain.

\section{Appendix A}

In this appendix we report a detailed comparison between the approximations $\tilde{k}, \tilde{g} v_{D}$ and $\tilde{g} v_{I}$ developed in Section 2 and 3 and the
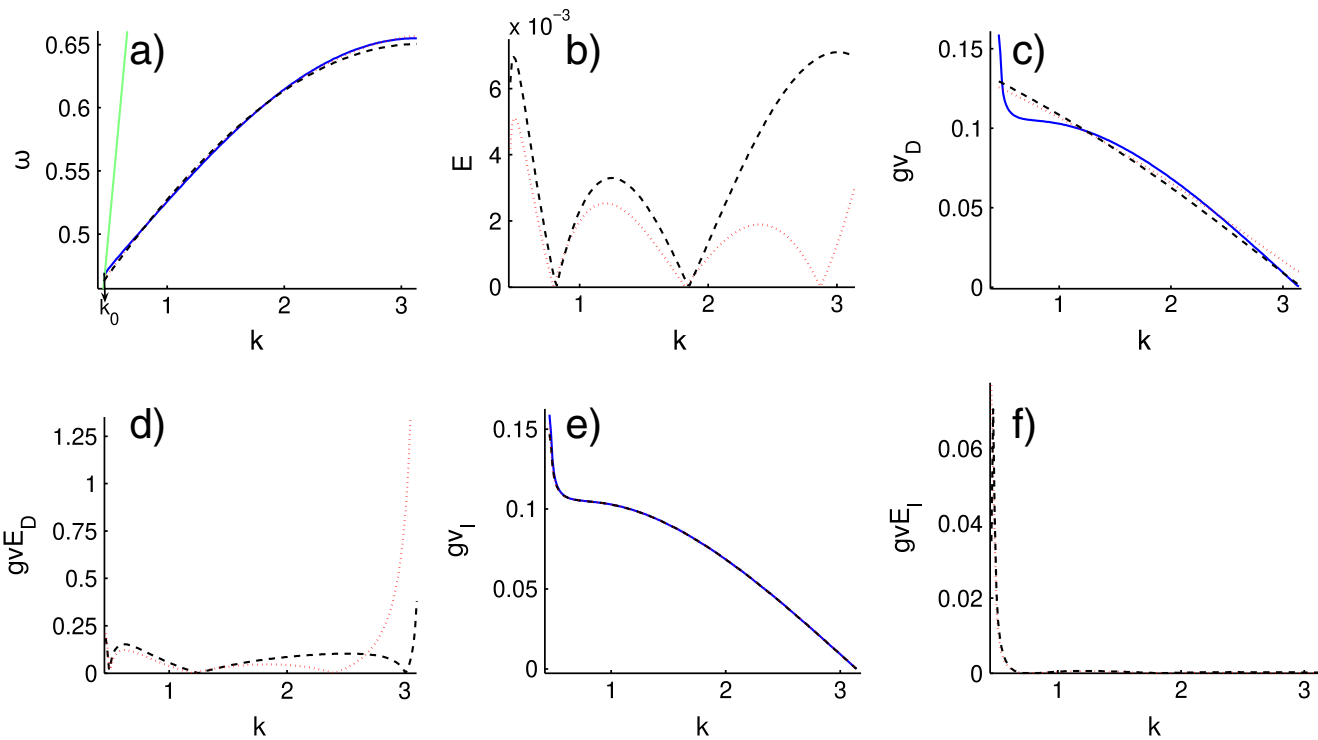

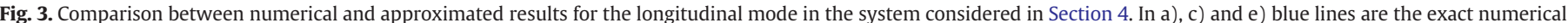

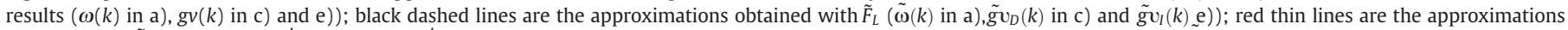

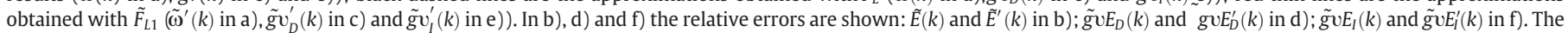
green line in a) is the light line, while $k_{o}$ is the point where $\omega(k)$ come off the light line. 

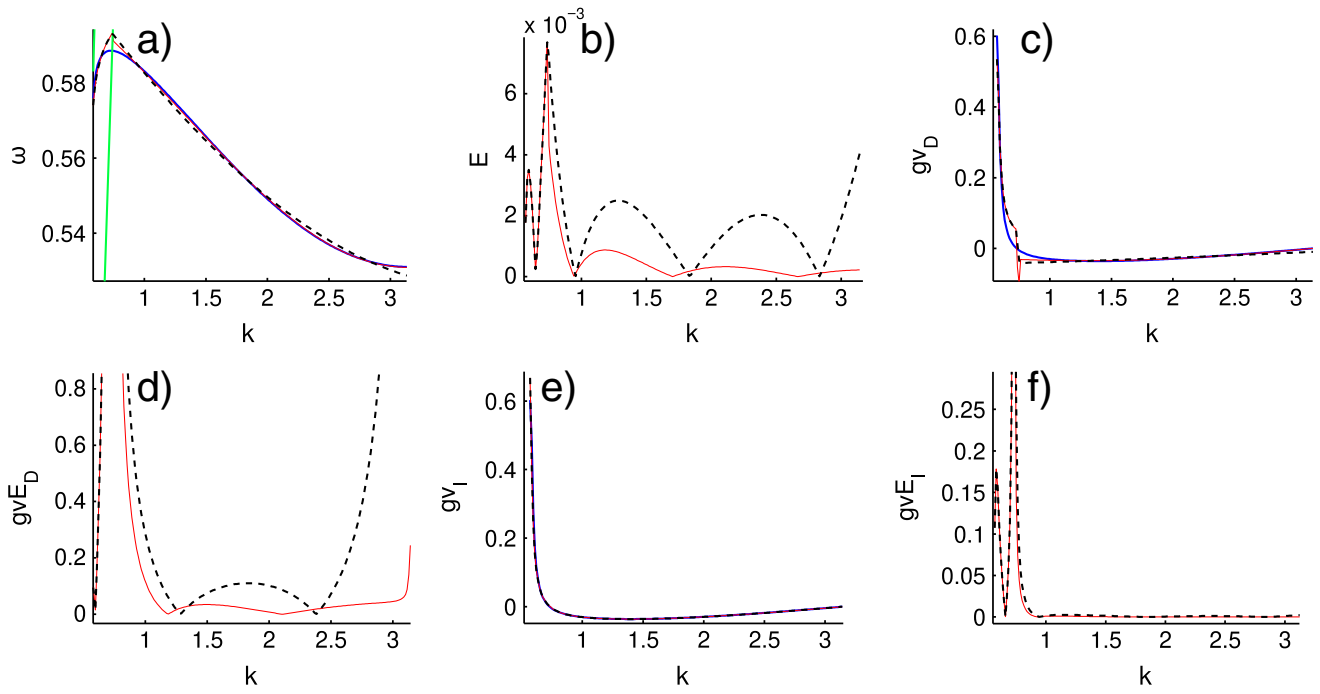

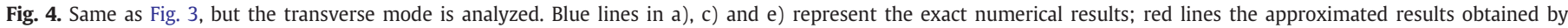
interpolating $F_{T}$ with $F_{T 1}$ in $R_{T 1}$ and $F_{T 2}$ in $R_{T 2}$. Region $R_{T 1}$ is between the two green lines in a), the first of whom on the left is the light line.

exact values $k$ and $g v$ obtained by solving numerically Eqs. (1) and (2). We refer to the system analyzed in the previous section. Relation $k(\omega)$ is not a function, because for any $\omega$ more than one $k$ can exist, while on the contrary $\omega(k)$ is a function. Then, instead of evaluating the difference $\tilde{k}(\omega)-k(\omega)$ at a fixed $\omega$, we evaluate the difference $\omega(k)-\tilde{\omega}(k)$ at a fixed
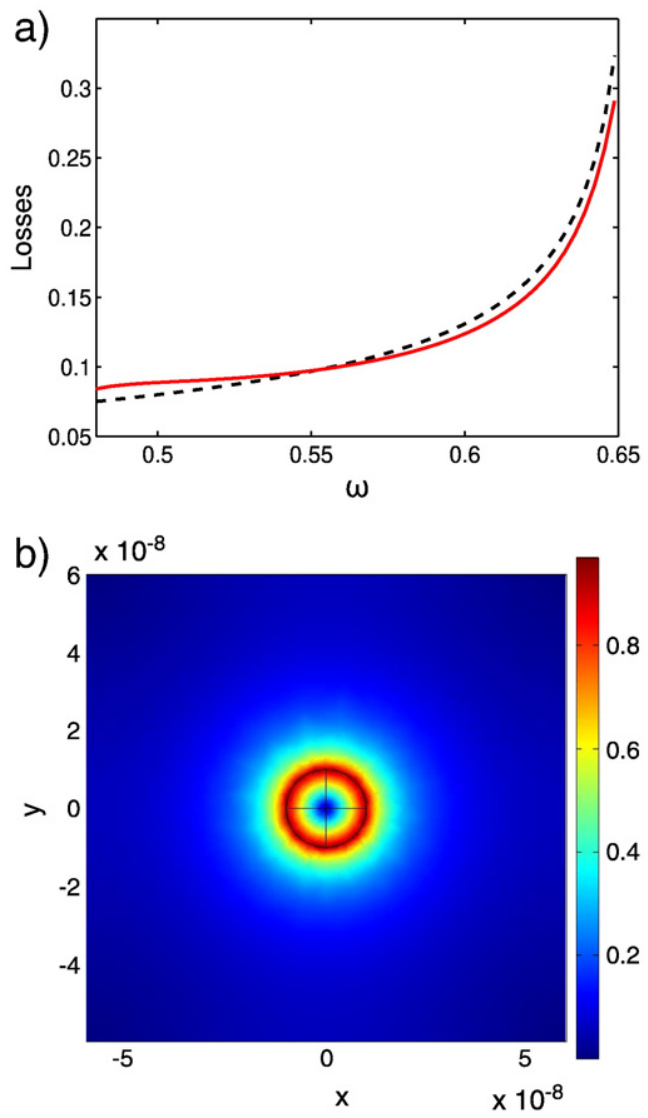

Fig. 5. In a) the comparison is shown between losses $\gamma_{L}$ calculated as imaginary part of $k$ obtained by numerically solving Eq. (2) (red lines) and losses $\gamma_{P L}$ predicted by Eq. (18) (black dashed lines). In b) the longitudinal mode at $\omega=0.5$ is shown (norm of the magnetic field). $k$, where $\omega(k)$ and $\tilde{\omega}(k)$ are the inverse of $k(\omega)$ and $\tilde{k}(\omega)$. For the same reason we evaluate the group velocities as function of $k$ instead of $\omega$. The exact group velocity is calculated as $g v(k)=\omega^{(k)}(k)$, while $\tilde{g} v_{D}(k)=\tilde{\omega}^{(k)}(k)$ and $\tilde{g} v_{I}(k)$ is calculated from Eq. (5) using the estimation $\tilde{\omega}(k)$ as value of $\omega$. The errors are evaluated as:

$E(k)=\sqrt{\frac{(\tilde{\omega}(k)-\omega(k))^{2}}{\omega(k)^{2}}}$

$\hat{E}=\int_{k=k 0}^{\pi} E(k)$

$g v E_{D, I}(k)=\sqrt{\frac{\left(\tilde{g} v_{D, I}(k)-g v(k)\right)^{2}}{g v(k)^{2}}}$

$g \hat{v} E_{D, I}=\int_{k=k 0}^{\pi} g v E_{D, I}(k)$

where $k_{0}$ in Eqs. (20) and (22) is the cut-off value (see Fig. 3a)). $E(k)$, $g v E_{D}(k)$ and $g v E_{I}(k)$ describe the relative error point-by-point in $k$, while $\hat{E}, g \hat{v} E_{D}$ and $g \hat{v} E_{I}$ represent their mean value.

Let us start by analyzing the results for the longitudinal mode, comparing the approximations done when $F_{L}$ is interpolated by $\tilde{F}_{L}$ and by $\tilde{F}_{L 1}$. We indicate with the superscript ' the results obtained when interpolating with $\tilde{F}_{L 1}$, without any superscript those obtained with $\tilde{F}_{L}$. Eq. (3) is used in order to estimate $k$. In Fig. 3a) the functions $\omega(k), \tilde{\omega}(k)$ and $\tilde{\omega}^{\prime}(k)$ and in Fig. 3b) the errors $E(k)$ and $E^{\prime}(k)$ are shown. It can be seen that the approximations are very good, as confirmed by the mean errors $\hat{E}$ and $\hat{E}^{\prime}$ that are respectively $0.5 \%$ and $0.2 \%$. The approximation $\tilde{\omega}^{\prime}(k)$, almost equal to $\omega(k)$ (see Fig. 3a)), is a bit better because the interpolation region $R_{L 1}$ is smaller than $R$; we note in Fig. $3 b$ ) that the errors in both cases are higher near the light line and close to $k=\pi$, i.e. the zones where the interpolation of $F_{L}$ is more difficult. In Fig. 3c) and in e) there is the comparison between the group velocities calculated in the direct and the indirect way, while in Fig. 3d) and in f) the corresponding errors. As we expected the indirect estimations $\tilde{g} v_{I}(k)$ and $\tilde{g} v_{I}^{\prime}(k)$ are better than the direct ones: the mean errors are $g \hat{v} E_{D}=8.8 \%, g \hat{v} E_{D}^{\prime}=7.7 \%$, gv̂E $E_{I}=1.3 \%$, $g \hat{v} E_{I}^{\prime}=1.4 \%$.

We move now to the description of the transverse case, whose dispersion curve stays in regions $R_{T 1}$ and $R_{T 2}$, so that Eqs. (8) and (9)-(10) are respectively used in order to estimate $k$. In Fig. $4 \mathrm{a}), \mathrm{b}), \mathrm{c}$ ) and d) it can 
be seen that there is a very good agreement between $\tilde{\omega}(k)$ and $\omega(k)$ in $R_{T 2}$ $(\hat{E}=0.06 \%)$ and in fact even the derivative $\tilde{g} v_{D}(k)$ matches quite well the group velocity $g v(k)$ : the error $g \tilde{v} E_{D}(k)$ in $R_{T 2}$ has a mean value $g \hat{v} E_{D}=14.8 \%$ but it is almost concentrated near $k=0.75$, where the group velocity aims at zero. These are good results especially considering that the group velocity in $R_{T 2}$ is very small, so that it is difficult to detect the little slope variations of $\omega(k)$. Fig. 4e) and f) describe $\tilde{g} v_{I}(k)$, that approximate quite perfectly $g v(k)\left(g \hat{v} E_{I}=0.6 \%\right.$ in $\left.R_{T 2}\right)$ : the relative error $g v E_{I}(k)$ is high (near $25 \%$ ) only near $k=0.75$ because at this point the group velocity is almost zero. Note also that in $R_{T 1}$ errors are higher than in $R_{T 2}\left(\hat{E}=0.3 \%, g \hat{v} E_{I}=11.7 \%\right)$ and that in proximity of the line that divide $R_{T 1}$ from $R_{T 2}$, around $k=0.74, \tilde{g} v_{D}(k)$ is discontinuous, that is due to the different estimations used in $R_{T 1}$ and $R_{T 2}$, while this is not a problem with the indirect estimation $\tilde{g} v_{I}(k)$, as it can be seen in Fig. $\left.4 \mathrm{e}\right)$.

We conclude by noting that the dispersion curves of this chain stay in the low part of region $R\left(R_{T 2}\right)$, but similar results have been obtained when considering other systems whose dispersion curves stay in the middle $\left(R_{T 3}\right)$ and upper $\left(R_{T 4}\right)$ part of $R$, thus validating the approximations proposed in the previous sections.

\section{References}

[1] M. Quinten, A. Leitner, J.R. Krenn, F.R. Aussenegg, Opt. Lett. 23 (1998) 1331.

[2] M.L. Brongesma, J.W. Hartman, H.A. Atwater, Phys. Rev. B 62 (2000) R16356.

[3] S.A. Maier, P.G. Kik, A. Harry, Phys. Rev. B 67 (2003) 205402.

[4] W.H. Weber, G.W. Ford, Phys. Rev. B 70 (2004) 125429.

[5] J.N. Anker, W.P. Hall, O. Lyanders, N.C. Shan, J. Zhao, R.P. Van Duyne, Nat. Mater. 7 (2008) 442.

[6] E. Ozbay, Science 311 (2006) 189.

[7] N. Fang, et al., Science 308 (2005) 534.

[8] H. Atwater, A. Polman, Nat. Mater. 9 (2010) 205.

[9] J.M. Gerardy, M. Ausloos, Phys. Rev. B 25 (1982) 4204.

[10] D.S. Citrin, Nano Lett. 4 (2004) 1561.

[11] M. Conforti, M. Guasoni, J. Opt. Soc. Am. B 27 (2010) 1576 